\title{
Erratum to: Reproductive isolation between Stigmaeopsis celarius and its sibling species sympatrically inhabiting bamboo (Pleioblastus spp.) plants
}

\author{
Younghae Chae $\cdot$ Nanako Yokoyama $\cdot$ Katsura Ito $\cdot$ Tatsuya Fukuda \\ Ryo Arakawa $\cdot$ Yan-Xuan Zhang $\cdot$ Yutaka Saito
}

Published online: 11 February 2015

(C) Springer International Publishing Switzerland 2015

\section{Erratum to: Exp Appl Acarol \\ DOI 10.1007/s10493-014-9865-0}

Due to an unfortunate turn of events, errors were introduced in the tables of the above mentioned publication. In Table 1, the dates in the fourth column (Date) were not fully specified. The footnote in Table 2 contained incorrect information as only Tukey's multiple comparison test was used and not Ryan's procedure. The data in Table 3, however, were created using Ryan's multiple comparison test instead of Tukey's. The correct representation of all three tables is published on the following pages and should be treated as definitive by the reader.

The online version of the original article can be found under doi:10.1007/s10493-014-9865-0.

Y. Chae $\cdot$ N. Yokoyama $\cdot$ K. Ito $(\bowtie) \cdot$ T. Fukuda $\cdot$ R. Arakawa $\cdot$ Y.-X. Zhang $\cdot$ Y. Saito Laboratory of Insect Ecology, Faculty of Agriculture, Kochi University, 200 Monobeotsu, Nankoku, Kochi 783-8502, Japan e-mail: ktr@kochi-u.ac.jp 


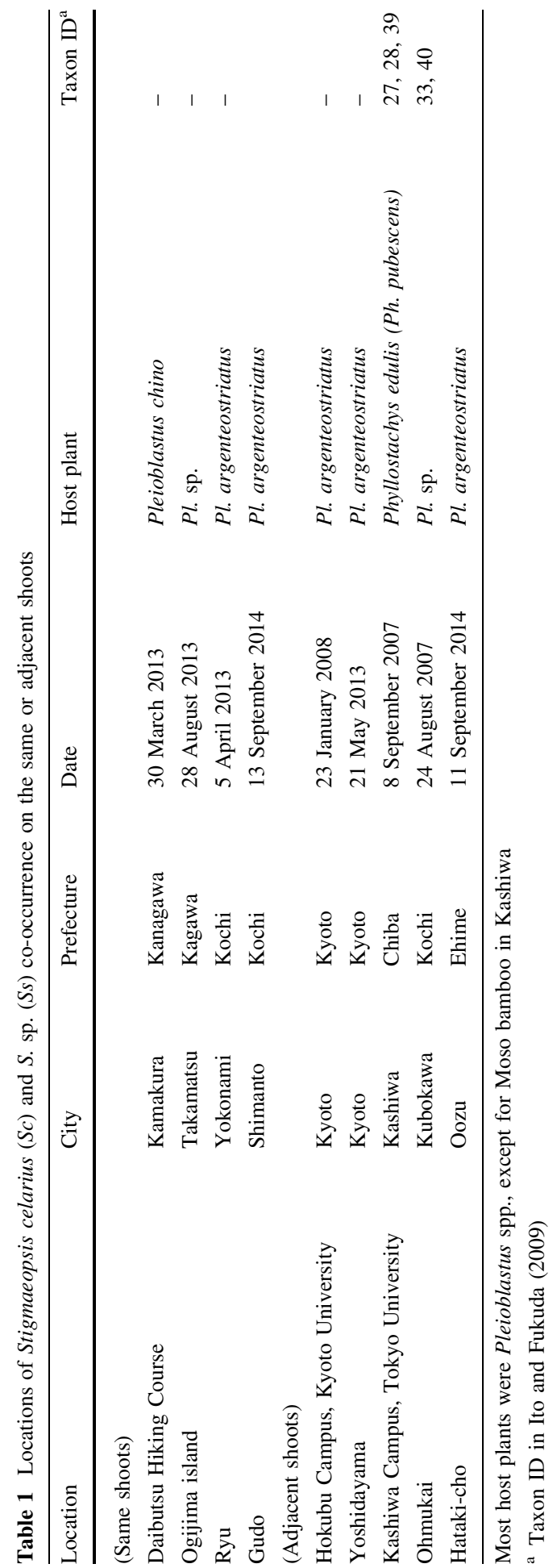


Table 2 Summary of reciprocal crosses between Stigmaeopsis celarius $(S c)$ and $S$. sp. (Ss). Proportion of success of male intrusion into the female nest, the number of eggs laid in 14 days, and the sex ratio of offspring (the proportion of males to the total offspring) are presented (Mean \pm SD)

\begin{tabular}{|c|c|c|c|c|}
\hline Crossing & $\mathrm{n}$ & Prop. intrusion & No. eggs & Prop. males \\
\hline$S c+\times S c$ o & 15 & 1.0 & $12.13 \pm 5.17^{\mathrm{a}}$ & $0.13 \pm 0.09^{\mathrm{a}}$ \\
\hline$S c q \times S s \widehat{\jmath}$ & 18 & 1.0 & $1.89 \pm 1.18^{\mathrm{b}}$ & $1.00 \pm 0.00^{\mathrm{b}}$ \\
\hline$S s$ 우 $\times S c \hat{\sigma}$ & 16 & 1.0 & $1.88 \pm 1.50^{\mathrm{b}}$ & $1.00 \pm 0.00^{\mathrm{b}}$ \\
\hline$S s q \times S s \hat{\jmath}$ & 17 & 1.0 & $9.18 \pm 1.13^{\mathrm{c}}$ & $0.19 \pm 0.10^{\mathrm{a}}$ \\
\hline
\end{tabular}

Significant differences within a column are indicated by different letters (Tukey's multiple comparison test, $P<0.05)$

Table 3 Parameters of copulatory behavior in reciprocal crosses between Stigmaeopsis celarius $(S c)$ and $S$. sp. $(S s)$

\begin{tabular}{|c|c|c|c|c|c|c|c|c|}
\hline \multirow[t]{2}{*}{ Cross } & \multicolumn{2}{|c|}{$\begin{array}{l}\text { (i) Prop. } \\
\text { copulation }^{\dagger}\end{array}$} & \multicolumn{2}{|c|}{$\begin{array}{l}\text { (ii) No. of copulation } \\
\text { attempts }\end{array}$} & \multicolumn{2}{|c|}{$\begin{array}{l}\text { (iii) Copulation } \\
\text { period }(s)^{+}\end{array}$} & \multicolumn{2}{|c|}{$\begin{array}{l}\text { (iv) Time for mounting } \\
\text { (s) }\end{array}$} \\
\hline & $\mathrm{n}$ & $\%$ & $\mathrm{n}$ & Mean \pm SD & $\mathrm{n}$ & Mean \pm SD & $\mathrm{n}$ & Mean \pm SD \\
\hline$S c+\propto S c \hat{\jmath}$ & 13 & $0.62^{\mathrm{ab}}$ & 13 & $0.6 \pm 0.5^{\mathrm{a}}$ & 8 & $89.5 \pm 48.6^{\mathrm{a}}$ & 8 & $4,829 \pm 4,370^{\mathrm{a}}$ \\
\hline$S c \rho+S s_{\varnothing} \widehat{ }$ & 13 & $0.23^{\mathrm{a}}$ & 13 & $1.4 \pm 3.2^{\mathrm{a}}$ & 3 & $45.7 \pm 16.5^{\mathrm{a}}$ & 3 & $2,688 \pm 1,065^{\mathrm{ab}}$ \\
\hline$S s_{\uparrow} \times S c_{\circlearrowleft}^{\widehat{\sigma}}$ & 15 & $0.67^{\mathrm{ab}}$ & 15 & $10.4 \pm 13.2^{\mathrm{a}}$ & 10 & $0.0 \pm 0.0^{\mathrm{b}}$ & 10 & $929 \pm 1,825^{\mathrm{b}}$ \\
\hline$S s_{+} \times S s_{\bigcirc}^{\widehat{0}}$ & 14 & $0.86^{\mathrm{b}}$ & 14 & $1.4 \pm 1.1^{\mathrm{a}}$ & 12 & $80.5 \pm 76.5^{\mathrm{a}}$ & 12 & $5,253 \pm 4,486^{\mathrm{a}}$ \\
\hline
\end{tabular}

$n$ Indicates the number of males in each cross. (i) Proportion of males showing copulatory behavior (opisthosoma raising), (ii) the number of copulation attempts by males, (iii) copulation period of copulating males, and (iv) time required for pre-copulatory mounting from first contact with a female

Significant differences within a column are indicated by different letters:

$\dagger$ Ryan's multiple comparison test for proportion data $(P<0.05)$

\$ Steel-Dwass test $(P<0.05)$ 